\title{
LITERATURWISSENSCHAFT
}

http://dx.doi.org/10.18778/2196-8403.2007.09

\author{
KATARZYNA DZIKOWSKA
}

\section{Ein Zeichen für den Nächsten. Emmanuel Levinas liest Paul Celan}

Paul Celan i Emmanuel Levinas zapewne nigdy się osobiście nie spotkali, pomimo iż przez wiele lat mieszkali niedaleko siebie, w tej samej dzielnicy Paryża. Można jednak mówić o spotkaniu ich myślenia, zarówno na płaszczyźnie filozofii, jak i literatury - w refleksji o człowieku i dziele literackim, przy czym obie te płaszczyzny w sposób oczywisty splatają się ze sobą. Niniejszy artykuł podejmuje próbę przyjrzenia się temu spotkaniu z perspektywy pism Emmanuela Levinasa, jego eseju poświęconego poezji Celana i należącego do jego fundamentalnych tekstów filozoficznych Inaczej niż być lub poza istota.

Paul Celan und Emmanuel Levinas begegneten einander höchstwahrscheinlich nie, obwohl sie mehrere Jahre lang in Paris nicht weit voneinander entfernt wohnten. Man kann aber von einer Begegnung ihres Denkens sprechen - sowohl auf der Ebene der Philosophie als auch auf der Ebene der Literatur, in der Reflexion über den Menschen und über das Wesen des literarischen Werkes, wobei die Überschneidung der beiden Ebenen selbstverständlich ist. Der vorliegende Beitrag stellt den Versuch dar, diese Begegnung aus der Perspektive zweier Schriften von Levinas näher zu betrachten: seines Essays über die Dichtung Paul Celans und eines seiner fundamentalen philosophischen Werke, Jenseits des Seins oder anders als Sein geschieht.

Paul Celan and Emmanuel Levinas have most never met, although they lived in the same quarter in Paris for many years. But we may speak of an encounter of their thinking - philosophical and ,philological', about man and about the essence of literature. It is evident that both kinds of thinking deeply coincide. The article tries to look upon this encounter from the point of view of two writings of Emmanuel Levinas: his essay on the poetry of Paul Celan and one of his basic philosophical written studies, Autrement qu' être ou au-delà de l'essence. 


\section{Ein Philosoph, ein Dichter, eine Schicksalsgemeinschaft}

Über zwanzig Jahre lang lebten sie in Paris, davon fast fünfzehn in demselben ,vornehmen' Viertel - Quartier XVI - am rechten Ufer der Seine. Höchstwahrscheinlich sind sie einander nie persönlich begegnet. Emmanuel Levinas und Paul Celan, ein aus Litauen stammender Philosoph und ein in Rumänien geborener Dichter. Beide Söhne des auserwählten Volkes, wobei man nur den ersten einen treuen Sohn des Alten Bundes nennen kann. Levinas war nicht nur gläubiger Jude, sondern auch Talmudgelehrter. Celan schien der festen Überzeugung zu sein, der Gott seiner Väter sei nicht sein Gott, obwohl er in seinen Gedichten nicht selten präsent ist. Emmanuel Levinas und Paul Celan - ihren Geburtsdaten nach trennt sie kaum eine Generation - verbindet jedoch eine Schicksalsgemeinschaft. Es ist diese Schicksalsgemeinschaft, die alle Juden vereinigt, die im Europa der ersten Hälfte des 20. Jahrhunderts gelebt haben: die Erfahrung des deutschen Nazismus. Die ganze Familie von Levinas, die in Kaunas lebte, wurde von den Nazis ermordet, er selbst überlebte den Krieg in einem Stalag. Celans Eltern wurden in einem Arbeitslager ermordet, er selbst hatte ein ähnliches Lager überlebt, aber eben die Tatsache, dass er überlebt hatte, wurde zu einem Trauma für sein ganzes Leben. Aber es gibt noch eine andere Levinas und Celan verbindende Erfahrung. Es ist der Zerfall der alten europäischen Ordnung infolge des Ersten Weltkrieges und die Bedrohung durch den Bolschewismus. Die Geschichte von Kaunas, jener Stadt, in der Levinas 1905 geboren wurde, wurde durch immer wieder neue Besatzer geschrieben. Es war zuerst die russische Herrschaftszeit (Folge der Teilung Polens unter die drei Mächte Preußen, Österreich-Ungarn und eben das zaristische Russland), seit 1915 die deutsche Okkupation. Zwar wurde Kaunas nach 1918 zu einer Stadt des unabhängigen Litauen, aber schon 1940 kam wieder die russische, diesmal in Form der bolschewistischen Besatzung. Erst 1990 ist Litauen aus der Sowjetunion ausgetreten und zum souveränen Staat geworden. Paradoxerweise wurde gerade Russisch zur Muttersprache von Levinas. Russisch war die Sprache, die er unter seinen Nächsten sprach, vor allem mit der Ehefrau. Die Sprache seiner Philosophie war aber das Französische. Paul Celan wurde 1920 in die untergehende Welt der k.u.k.-Monarchie hineingeboren. Zwar existierte sie in Celans Geburtsjahr nicht mehr als ein Staatsgebilde; was aber immer noch eine starke Wirkung ausübte, war das kulturelle Erbe, mit dem sich die Mehrheit der in den Gebieten des alten Kakaniens lebenden Juden identifizierte. Es ist eine Anmerkung mit anekdotischem Charakter, aber doch in dem Kontext nennenswert: Für Levinas war Russisch die Muttersprache, und Celan sang, nach dem Zeugnis seiner 
Freunde, mit großer Vorliebe russische Lieder (CHALFEN 1983:107; BöTTIGER 1996:19). Das ändert jedoch überhaupt nichts an der Tatsache, dass seine Muttersprache Deutsch war. Deutsch war die Sprache, die er nie aufgab, obwohl es auch die Sprache der Mörder seiner Eltern und seines Volkes war. Die Heimatstadt Celans, Czernowitz, lag in der Bukowina, einem Teil von Österreich-Ungarn, der nach seinem Zerfall Rumänien zufiel und später, seit 1940, zur Sowjetunion gehörte. Dann kam die Besatzung durch Hitlerdeutschland und 1944 erneut die sowjetische Regierungsordnung. Die Befreiung der Heimat seiner Kindheit und Jugend vom Totalitarismus im Jahre 1990 erlebte Celan nicht mehr - zwanzig Jahre zuvor hatte er in Paris Selbstmord begangen.

Das Problem der Heimat, mit der man die frühesten Jahre des Lebens, die Kindheit und die Jugendzeit verbindet, war für Levinas wie für Celan mit Sicherheit kein einfaches. Man muss hier auf einige spezifische Differenzen hinweisen. Levinas war stets darauf bedacht, dass man seinen Familiennamen in Frankreich ohne Akzent schreibt, als einen jüdisch-litauischen. Er selbst schrieb ihn so; auch in den meisten Büchern, die zu seinen Lebzeiten veröffentlicht wurden, befolgt man diese Schreibweise (ziemlich unverständlich ist also die insbesondere im deutschsprachigen Raum verbreitete Schreibweise mit dem Akzent). Anders Celan. Sein Familienname lautete Antschel (in der deutschen Schreibweise; die rumänische war Ancel); Celan ist ein Anagramm, das er seit 1948 als seinen Namen benutzte. Beide waren keine Exilanten, wie man es manchmal fälschlicherweise behauptet. Frankreich wurde für beide zu einem Zuhause, für das sie sich frei entschlossen hatten, beide wurden zu französischen Staatsbürgern. Die Gründe für diese Entscheidung waren jedoch jeweils andere. Für Levinas war es eine Wahl des Herzens, für Celan die Folge der Unfähigkeit, sich in der geistigen Atmosphäre im Österreich der Nachkriegszeit zurechtzufinden. Ein weiterer Unterschied betraf ihr Verhältnis zu Deutschland. Celan fühlte sich ständig durch dieses Land angezogen, beinahe angelockt, auch wenn jede Reise dorthin, jeder Besuch mit einem erneuten Leiden des sensiblen Dichters verbunden war. Was ihm immer wieder wehtat, war jeder, auch der kleinste Vorfall antisemitischen Charakters. Auch durch die Plagiatanschuldigungen (die sogenannte Goll-Affäre ${ }^{1}$ ), an die sich manche

1 Yvan Goll war ein in Paris lebender jüdischer Dichter, dessen französische Gedichte Celan auf seinen Wunsch ins Deutsche übertragen hat. Nach dem Tode von Goll im Jahre 1950 hat jedoch seine Frau, Claire Goll, in mehreren Aussagen Plagiatvorwürfe gegenüber Celan erhoben. Die drei übersetzten Gedichtbände sind unveröffentlicht geblieben. Obwohl die Behauptungen von Claire Goll völlig haltlos waren, ist für Celan diese regelrechte Kampagne gegen ihn zu einem 
deutsche Kritiker und Literaturwissenschaftler anschlossen, fühlte er sich zutiefst verletzt. Das alles wurde für ihn zu einer weiteren traumatischen Erfahrung. Trotz alldem suchte Celan gerade in Deutschland seine Leser, und von dort erwartete er Verständnis und Anerkennung. Levinas behauptete zwar, wer in der Philosophie Erfolg haben wolle, müsse zuerst im deutschsprachigen Raum Anerkennung finden, nach den Schrecken des Holocaust legte er jedoch ein Gelübde ab: Sein Fuß solle nie wieder den deutschen Boden betreten - und er hielt sich konsequent an dieses Gelübde. Emmanuel Levinas blieb jedoch der deutschsprachigen Kultur, insbesondere der Philosophie gegenüber nicht gleichgültig. Er nahm an Symposien teil, die von deutschen akademischen Institutionen veranstaltet wurden, traf sich mit deutschen Forschern und Kennern seiner Philosophie, gab Interviews. Wenn er aber in seinen Äußerungen (in philosophischen Arbeiten wie auch in Gesprächen) Dichter zitierte, so waren es meistens die Klassiker der russischen Literatur, manchmal Shakespeare, selten Goethe, obwohl er mit der deutschsprachigen Literatur seit seiner Jugend vertraut war.

Im Jahre 1975 erschien Levinas' Buch Eigennamen (frz. Noms propres). Es ist eine Sammlung verschiedener Essays, die in einem Zeitraum von mehreren Jahrzehnten entstanden. Diese Essays sind sowohl der Philosophie als auch der Literatur gewidmet. Darunter befindet sich eine kleine Studie zu Paul Celan. In der Einführung zu der Sammlung schreibt LEVINAS (1988:9):

Eigennamen, deren ,Aussage' ein Gesicht bedeutet, Eigennamen sind unter allen Namen und Gemeinplätzen diejenigen, die der Auflösung des Sinns widerstehen und uns helfen zu sprechen. Erlauben sie uns nicht, hinter brïchigen Aussagen zwar das Ende der einen Verstehbarkeit (Intelligibilität), aber auch den Morgen einer anderen zu erahnen? Was zu Ende geht, ist vielleicht die ausschließlich an das vom Wort getragene Sein, die an das Ausgesagte des Aussagens gebundene Rationalität. Gebunden an ein Gesagtes, das Wissensgüter und Wahrheit als unveränderliche Identitäten vermittelt, sich der sich selbst genügenden Identität eines Seins oder Systems eingliedert, abgeschlossen, perfekt; das Differenzen, die es verraten oder beschränken könnten, ablehnt oder absorbiert. Jene Verstehbarkeit, die ihren Höhepunkt in der von Hegel (bei dem vermutlich die Philosophie des Selben und die Immanenz bzw. die Ontologie ihren Abschluß finden) behaupteten letzten Identität des ,Identischen und des Nichtidentischen“ erreicht.

Levinas' einführenden Worten nach haben die Personen, auf die er sich in seinen Essays bezieht (neben Paul Celan sind dies unter anderen auch Maurice Blanchot, Marcel Proust, Martin Buber), eine ganz konkrete Aufgabe zu er-

Trauma geworden, von dem er sich bis an sein Lebensende nicht hat befreien können (FELSTINER 2000:93f., 107, 204-206, 288). 
füllen, oder man dürfte mindestens hoffen, dass sie mit ihren Werken zur Entwicklung einer neuen Denkart beitragen könnten. Levinas spricht darüber mit Hilfe von Termini, die für seine Philosophie typisch sind: vom Ungenügen der Ontologie, vom Ungenügen der Sprache als des Gesagten, das heißt des in sich selbst Verschlossenen, auch dem anderen Menschen gegenüber verschlossen (mit den Begriffen von Martin Buber ausgedrückt - monologisch). LEviNAS (1988:7) weist mit einer entschlossenen Eindeutigkeit auf die Gründe eines solchen Zustands hin: „Zwei Weltkriege, dazu lokale Kriege, der Nationalsozialismus, der Stalinismus, dazu die Entstalinisierung, Lager, Gaskammern, Atomwaffenarsenale, Terrorismus und Arbeitslosigkeit - recht viel für eine einzige Generation, und hätte sie all dies auch nur als Zuschauer erleben müssen." So lautet der erste Satz der Einführung zu Eigennamen. Dieser Gedanke wird in einem anderen Essay dieser Sammlung, betitelt Namenlos, wiederkehren: „Seit Ende des Krieges hat das Blut nicht aufgehört zu fließen. Rassismus, Imperialismus und Ausbeutung sind so erbarmungslos wie je. Nationen und Menschen geben sich $\mathrm{Haß}$ und Verachtung hin, fürchten Elend und Zerstörung." (LEVINAS 1988:101) Es sind durchaus harte Worte. Aber es sind leider auch wahre Worte. Man hört in ihnen den ganzen Schmerz desjenigen, der überlebte, der aber sieht, dass die Menschen nichts lernten. Es ist auch der Schmerz desjenigen, der sich von der schrecklichen Erinnerung nicht befreien kann, der sich des grausamen Schicksals, das seine Nächsten getroffen hat, immer wieder aufs Neue bewusst werden muss. Und trotz alldem bemüht er sich, Schlüsse zu ziehen und den jüngeren Generationen Wahrheiten zu zeigen, die notwendig sind, um eine neue Wirklichkeit aufzubauen. Levinas spricht als Jude zur jüdischen Jugend. Er spricht von drei Wahrheiten, die seiner Meinung nach aus der Erfahrung des Holocaust zu lernen sind.

Um wie Menschen zu leben, bedürfen die Menschen unendlich viel wenigerer Dinge als die großartigen Kulturen, die sie bewohnen - das ist die erste Wahrheit. [...] Wir befinden uns wieder in der Wüste, einem landschaftslosen, oder, wie das Grab, nur für uns geschaffenen Raum; wir sind in den Raum, der ein Behältnis ist, zurückgekehrt. Das Ghetto ist auch dies - und nicht nur Abgetrenntsein von der Welt. Aber - zweite Wahrheit - und auch sie fällt mit einer uralten Sicherheit und einer uralten Hoffnung zusammen - in den entscheidenden Augenblicken, in denen sich die Hinfälligkeit so vieler Werte erweist, besteht die menschliche Würde allein darin, an ihre Rückkehr zu glauben. Wenn , alles erlaubt ist', besteht die höchste Pflicht darin, sich schon für diese Werte der Freiheit verantwortlich zu fühlen. [...] Aber - die dritte Wahrheit - bei der unvermeidlichen Wiederaufnahme von Kultur und Assimilation müssen wir nun die neuen Generationen die nötige Kraft lehren, um in der Isolierung stark zu sein, und alles, was ein gefährdetes Bewußtsein aufzubewahren aufgerufen ist. Wir müssen, indem wir das Gedächtnis derjenigen bewahren, die, Juden oder Nichtjuden, ohne sich zu kennen 
oder auch nur zu sehen, sich im größten Chaos so zu verhalten wußten, als ob die Welt nicht aus den Fugen wäre. [...] Wir müssen, durch solche Erinnerung hindurch, einen neuen Zugang zu den jüdischen Texten schaffen und dem inneren Leben aufs neue Priorität verleihen. Das ,innere Leben “ - angesichts so vieler Objektivismen und Realismen schämt man sich geradezu, ein so lächerliches Wort zu gebrauchen. (LEVINAS 1988:103f.)

Diese Worte wurden zum ersten Mal im Jahr 1966 veröffentlicht. Dadurch jedoch, dass der Essay Namenlos an die Sammlung Eigennamen angeschlossen wurde, als ihr abschließender Text, gewinnen sie wieder an Bedeutung und schaffen einen bestimmten Kontext für die ganze Sammlung. Im Lichte von Namenlos soll man die herbeigerufenen Personen und ihre Texte betrachten auch Paul Celan und seine Dichtung. Diesen Kontext bildet die Suche nach einem neuen Verständnis und nach Begegnungsorten, es ist eine Forderung nach der Verantwortlichkeit für die Werte, die für die Wahrung der Menschenwürde unentbehrlich sind, es ist auch ein Protest gegen eine Welt, in der alles erlaubt ist. Wenn Levinas von einer Welt sprach, in der alles erlaubt ist, hatte er zweifelsohne den bekannten, von ihm selbst des Öfteren zitierten Satz von Dostojewskij im Sinn: „Wenn es keinen Gott gibt, dann ist alles erlaubt.“ Der Protest gegen eine Welt, in der alles erlaubt ist, ist also zugleich ein Protest gegen eine Welt ohne Gott.

Im Jahre 1971 erschien in Paris eine kleine Auswahl aus Celans Werk: Strette. Poèmes suivis de Méridien et d'Entretiens dans la montagne. Es war eine zweisprachige, deutsch-französische Edition. Die Übersetzungen wurden noch von Celan selbst autorisiert. Der Band beinhaltet, wie schon der Titel besagt, eine Gedichtauswahl und zwei Prosatexte: Der Meridian - die Rede, die Celan anlässlich der Verleihung des Georg-Büchner-Preises am 22. Oktober 1960 gehalten hat, und Gespräch im Gebirg - entstanden im Jahre 1959 unter dem Einfluss der großen Enttäuschung, dass es nicht zu einer geplanten Begegnung des Dichters mit Theodor Adorno kam. Der Meridian ist keine konventionelle Dankrede. Es ist ein in lyrischer Prosa gehaltener Traktat über das Wesen des Gedichts. Gespräch im Gebirg, formal betrachtet auch ein lyrischer Prosatext, ist ein Dialog zweier Juden über die jüdische Identität. Beide Texte, sowohl Der Meridian als auch Gespräch im Gebirg, muss man als fundamentale Texte bezeichnen, wenn man begreifen will, wer Paul Celan war: vor allem Dichter und vor allem Jude. Nicht zuerst das eine und dann das andere. Für Celan waren dies zwei untrennbare Bestandteile seiner Identität. Auf diese beiden Texte, Der Meridian und Gespräch im Gebirg, und auf das Gedicht Engführung aus dem Band Strette ging Levinas in seinem Essay Vom Sein zum Anderen. Paul Celan ein. Der Essay wurde zum ersten Mal im Jahre 1972 veröf- 
fentlicht und dann im Jahre 1975 zum zweiten Mal in dem Band Eigennamen. Die selbständige Edition dieses Textes (Paul Celan. De l'être à l'autre) aus dem Jahre 2003 ist ein Hinweis darauf, dass ihm - sei es wegen des Interesses an Celan, sei es wegen Levinas oder der beiden - ein besonderer Platz zugewiesen wird.

Die Begegnung mit Celans Texten muss Levinas tief bewegt haben - sein Herz wie seinen Intellekt. Zwar blieb der Essay Vom Sein zum Anderen aus dem Jahre 1972 die einzige umfangreichere Äußerung des Philosophen zu Celan, man findet jedoch bei Levinas andere Spuren der Anerkennung dieses Dichters. Das vierte, Levinas' Worten nach für das Buch zentrale Kapitel (betitelt Die Stellvertretung, frz.: La Substitution) des Werkes Jenseits des Seins oder anders als Sein geschieht hat als Motto einen Vers aus einem von Celans Gedichten. Der Essay La Substitution entstand im Jahre 1967, und bei der ersten Edition aus dem Jahre 1968 fehlt dieses Motto noch. Es erschien erst in der Buchedition aus dem Jahre 1974. Es ist ein Vers aus dem Gedicht Lob der Ferne, entstanden im Jahre 1948: „Ich bin du, wenn ich ich bin“ (CELAN 1986/3:56). Levinas zitiert ihn in der Originalversion - auf deutsch. Denselben Vers zitiert Levinas erneut, ebenso auf deutsch, als eine gewisse Pointe zum Schluss seines Vortrags an der Sorbonne am 20. Februar 1976 (aus der Vortragsreihe Gott und die Onto-Theo-Logie). In der Buchedition beider Vortragsreihen, die Levinas 1975/1976 an dieser Universität hielt und die unter dem Titel Gott, der Tod und die Zeit erschien, trägt dieser Vortrag den Titel Die Subjektivität als An-Archie.

\section{Jenseits des Seins und das Wesen des Gedichts}

Levinas schrieb den Essay über Celan in einer zeitlichen Nähe zur Redaktion von Jenseits des Seins oder anders als Sein geschieht, eines seiner fundamentalen philosophischen Werke. Die meisten der Texte, die den Band bilden, auBer dem Kapitel Die Stellvertretung (La Substitution) aus dem Jahre 1968, entstanden im Zeitraum 1970-1972. Auch wenn die Idee des Buches und die erste Version auf das Ende der 60er Jahre zu datieren sind, so waren zweifelsohne die darauf folgenden Jahre eine Zeit intensiver Arbeit am ganzen Werk, das eben in Buchform erscheinen sollte. Während dieser Arbeit kam es zur Lektüre des Bandes Strette und dann zur Entstehung des Essays Paul Celan. Vom Sein zum Anderen. Schon der Titel des Essays knüpft an den Titel des Werkes Jenseits des Seins oder anders als Sein geschieht an. In dem abschließenden Kapitel schrieb LEVINAS (1998:393f.) zu seinem Buch: 
Dieses Buch interpretiert das Subjekt als Geisel und die Subjektivität des Subjekts als Stellvertretung, die mit dem sein des Seins - und des Seienden - bricht. [... Jedes Individuum ist gerufen,] aus dem Begriff des Ich aufzubrechen, aus seiner Ausdehnung in der Masse, im Volk, gerufen, aus Verantwortung zu antworten: ich, das heißt sieh mich, hier bin ich, für die Anderen, gerufen, in radikaler Weise den eigenen Platz oder den eigenen Schutz im Sein zu verlieren, einzutreten in die Allgegenwärtigkeit, die ebenso eine Utopie ist. Hier, sieh mich, für die Anderen Antwort außerhalb der Norm, deren Enormität oder Maßlosigkeit abgeschwächt wird durch den Verdacht der Heuchelei, der auftritt, sobald die Antwort mir zu Ohren kommt; zu Ohren, die vorgewarnt sind durch das sein des Seins, das heißt durch die Art, in der das Sein abläuft.

Man darf also sagen, dass im Titel des Celan-Essays die Hauptidee des Werkes Jenseits des Seins oder anders als Sein geschieht ausgedrückt wird: Nicht mehr das Sein des Ich steht im Zentrum des Interesses, sondern der andere Mensch. Deshalb bedeutet das Ich nicht mehr ein Sein, sondern die Verantwortlichkeit für den Anderen. Dieser sichtbare Zusammenhang berechtigt noch nicht zu der Feststellung, Levinas habe in Celans Texten eine Widerspiegelung seiner philosophischen Überlegungen gefunden. Man darf jedoch zwei Fragen stellen. Erstens: Ob die Lektüre von Celans Texten Bedeutung für die endgültige Formulierung der Hauptgedanken in Jenseits des Seins oder anders als Sein geschieht haben konnte, und wenn ja, welche? Zweitens: Ob man in Levinas' Essay über Celan Spuren der Hauptgedanken aus Jenseits des Seins oder anders als Sein geschieht finden kann, und wenn ja, welche? Dass man die erste Frage eher vorsichtiger, die zweite jedoch mit einer größeren Entschlossenheit stellen möchte, ergibt sich aus einer relativ einfachen Vermutung: Als wahrscheinlicher erscheint, dass Levinas die Texte von Celan aus der Perspektive des eigenen Denkens las, als dass die Lektüre von Strette einen bedeutenden Einfluss auf die Formulierungen seines philosophischen Werkes hätte ausüben können.

Das Gedicht [...] ein Zeichen für den Nächsten! Zeichen wofür? Für Leben? Wohlwollen? Komplizenschaft? Oder Zeichen für nichts, Komplizenschaft ohne Tat: Sagen ohne Aussage? Oder ein Zeichen, das sich selbst bezeichnet: das Subjekt zeigt das Zeichengeben an, bis es reines Zeichen wird. Elementare Kommunikation ohne Botschaft, lallende Kindheit des Diskurses, höchst ungeschicktes Eindringen in das berühmte ,Die Sprache spricht', Eintritt eines Bettlers in die ,Wohnung des Seins‘. (LEVINAS 1988:56)

Mit diesen Worten kommentiert Levinas den bekannten Satz aus dem Brief an Hans Bender, in dem Celan das Gedicht als Händedruck bezeichnete (CELAN 1986/3:177f.). Levinas kreist um den Begriff des Zeichens, als ob er die Definition des Gedichtes noch erweitern und präzisieren möchte. Eine ähnliche 
Äußerung, die beinahe wie ein Selbstzitat klingt, findet man in einem anderen Text von Levinas, im Kapitel Subjektivität und Unendlichkeit des Werkes Jenseits des Seins oder anders als Sein geschieht, in dem LEVINAS (1998:330f.) schreibt:

Bevor es sich in den Dienst des Lebens stellt als Austausch von Informationen durch ein linguistisches System, ist das Sagen Zeugnis, Sagen ohne Gesagtes, Zeichen an den Anderen. Zeichen wovon? Der Komplizenschaft? Einer Komplizenschaft ohne Absicht, einer Brüderlichkeit, einer Nähe, die nur möglich ist als ein Sich-Öffnen, als unvorsichtige Ausgesetztheit für den Anderen, rückhaltlose Passivität bis hin zur Stellvertretung und folglich als Aussetzung der Ausgesetztheit, eben als Sagen, Sagen, das nicht etwas sagt, das bedeutet, Sagen, das als Verantwortung die Bedeutung selbst ist, der Eine-für-den-Anderen, Subjektivität des Subjekts, das sich zum Zeichen macht, aber das man mißverstände, hielte man es für den stammelnden Ausdruck eines Wortes, denn es bezeugt die Herrlichkeit des Unendlichen.

Und in einem früheren Abschnitt desselben Kapitels liest man: „Als dem Anderen gegebenes Zeichen und schon Zeichen dieser Zeichengabe, als reines Bedeutungszeichen ist die Nähe keine Vermischung mit dem Anderen [...].“ (LEVINAS 1998:314) Zweifelsohne können und sollen diese Äußerungen gemeinsam gelesen werden. Aus einer solchen Zusammenstellung folgt, dass man das Gedicht als Nähe bezeichnen könnte. Das Gedicht ist Nähe. Das Gedicht ist Sagen. Das Gedicht ist Zeugnis. Dreimal ist die Rede von dem Zeichen, das dem anderen Menschen gegeben wird - nicht irgendeinem, nicht näher bestimmten Menschen, sondern einem Menschen, der als der Nächste bezeichnet wird. Wenn das Gedicht dem Nächsten gegenübersteht, ist es zugleich durch die Verantwortlichkeit für den Nächsten geprägt.

Es trifft sich also, daß sich für Celan das Gedicht genau auf jenem prä-syntaktischen und prä-logischen (wie das heute zweifellos unabdingbar ist), aber auch noch prä-dekuvrierenden Niveau bewegt: dem Moment des reinen Ergreifens, Drückens, das, vielleicht, eine Weise darstellt, die Hand selber mit zu geben. (LEVINAS 1988:57)

Und quasi parallel zu diesen Feststellungen liest man in Jenseits des Seins oder anders als Sein geschieht: „Das vor-ursprüngliche anarchische Sagen, das Nähe ist, Berührung, nicht endende Pflicht [...].“ (LevinAS 1998:351) Erneut weist eine solche parallele Lektüre auf eine sichtbare Nähe beider Texte hin, zugleich jedoch erlaubt sie es, das Verständnis vom Wesen des Gedichts zu vertiefen: Das Gedicht ist „Sagen“, „Berührung“, Teil einer Urordnung. Somit wird eine der Hauptthesen von Levinas' Philosophie herbeizitiert, die von der Erwählung des Menschen durch das Gute in der an-archischen Zeit, in einer 
Vorzeit, zur Verantwortlichkeit für den Anderen spricht. Eine Fortsetzung dieses Gedankens bildet Levinas' Kommentar, in dem er Celans Gedicht als ,eine der Frage vorausgehende, zuvorkommende Antwort“ (LEviNAS 1988:57) bezeichnet. Und wieder findet man in Jenseits des Seins oder anders als Sein geschieht eine genauso paradox klingende Feststellung, von einem „Gehorsam, der allem Hören des Gebots vorausgeht“" (LEVINAS 1998:325).

Levinas kommentiert und interpretiert die Texte von Paul Celan. Er tut es auf eine eigentümliche Art und Weise. Üblicherweise erwartet man von einem Kommentator und Interpreten eines dichterischen Textes, dass er die Sprache der Lyrik in eine nicht-lyrische, in gewissem Sinne epische Sprache übersetzt - als ob er nacherzählen und berichten wollte, was der Dichter auf seine eigene Art und Weise ausdrückt. Was in dem Essay Vom Sein zum Anderen vollzogen wird, ist keine Übersetzung von Celans Texten, sondern ein Gespräch mit ihnen, in dem sich Levinas auch einer stark metaphorisierten Sprache bedient. Er scheint die Gedanken, die er bei dem Dichter vorfindet, weiterzuführen und zu vertiefen. Wenn man den Stil aller Werke von Levinas berücksichtigt - in denen er sehr oft nach Metaphern greift - so muss man betonen, dass er auf keinen Fall die Sprache von Celan übernimmt. Dass bei der Lektüre des CelanEssays die Sprache des Dichters und die Sprache des Philosophen als sehr nahe verwandt erscheinen, lässt eine andere Verwandtschaft, eine geistige Verwandtschaft vermuten, deren Wurzel viel tiefer reicht als nur auf die Ebene der sprachlichen Ausdrucksmittel: Erst eine gemeinsame Lektüre des Essays Vom Sein zum Anderen und des Werkes Jenseits des Seins oder anders als Sein geschieht erlaubt ein tieferes Verständnis des ersteren. Man könnte hier vielleicht von einer spezifischen Form des hermeneutischen Zirkels sprechen spezifisch, weil man sich nicht zwischen einem Teil des Textes und seiner Ganzheit bewegt, sondern es mit einem komplizierten Prozess des EinanderDurchdringens und -Ergänzens der Gedanken zu tun hat, welcher sich zwischen mehreren Texten zweier Autoren verfolgen lässt.

Wenn man Levinas' Essay über Paul Celan analysiert, sollte man auf einige Bezüge zu zwei Philosophen hinweisen. Es sind Denker, mit denen Levinas an anderen Stellen wichtige Diskussionen führte, auch wenn sie auf verschiedenen Ebenen verliefen. Gemeint sind Martin Buber und Martin Heidegger. So schreibt LEVINAS (1988:58f.) in dem Celan-Essay:

Das Gedicht hält auf das ,Andere' zu. Es hofft, es befreit und verfügbar anzutreffen. Das einsame Werk des Dichters, der das wertvolle Wortmaterial meißelt, ist der Akt des ,Aufsuchens' eines ,Gegenüber'. Das Gedicht, wird Gespräch - oft ist es verzweifeltes Gespräch', ,[...] Begegnungen, Wege einer Stimme zu einem wahrnehmenden $\mathrm{Du}^{\prime}$ - die Buberschen Kategorien! Sollten sie etwa all den ge- 
nialen, vom geheimnisvollen Schwarzwald [dt. im Original] sich auf Hölderlin, Trakl und Rilke herabsenkenden Exegesen vorgezogen werden, um die Dichtung als Öffnerin der Welt und des Orts zwischen Himmel und Erde aufzuzeigen?

Levinas entdeckt in Celans Der Meridian Spuren der Philosophie von Martin Buber und polemisiert zugleich mit Heidegger. Dass Celan selbst beide Philosophien, sowohl Bubers als auch Heideggers, sehr gut kannte, dass er von Buber fasziniert, dagegen Heidegger gegenüber sehr kritisch eingestellt war - das alles wusste Levinas höchstwahrscheinlich nicht. Um so wichtiger erscheint seine Feststellung, für den Umgang mit Dichtung seien die Buberschen Kategorien besser geeignet als die Methoden Heideggers. Levinas argumentiert mit dem Hinweis: „Das Persönliche soll die Poesie des Gedichtes ausmachen. [...] Das Persönliche: von mir zum Anderen.“ (LEVINAS 1988:58) Die Polemik mit Heidegger, begonnen im Zusammenhang mit der Auffassung vom Wesen des Gedichts, verlagert sich jedoch auf eine außerliterarische Ebene. Zuerst schreibt LEVINAS (1988:61) noch über die Poesie:

Über das Fremdartige der Kunst und der Öffnung auf das Sein des Seienden hinaus geht das Gedicht einen Schritt weiter: das Fremdartige ist der Fremde oder der Nächste. Nichts ist fremdartiger und fremder als der Andere, und im Lichte der Utopie zeigt sich der Mensch. Außerhalb jeder Verwurzelung und Seßhaftwerdung: Heimatlosigkeit als Authentizität!

Dann geht er aber zu einem anderen Problem über: zum Problem der jüdischen Identität.

Das Überraschende an jenem Abenteuer, im Laufe dessen sich das Ich am NichtOrt dem Anderen widmet, ist jedoch die Rückkehr. [...] Als wäre das Zugehen auf den anderen mein Zusammentreffen mit mir und eine Einpflanzung in eine nunmehr heimatliche Erde, befreit vom ganzen Gewicht meiner Identität. [...] Heimat oder gelobtes Land? Speit sie ihre Bewohner aus, wenn sie die Kreisbahn, die ihnen diese Erde vertraut gemacht hat, ihr Umherirren vergessen, das keine Entfremdung von ihr, sondern Ent-heidnung war? Diese, durch das Sich-dem-Anderen-Zuwenden gerechtfertigte Heimat ist jedoch ihrem Wesen nach jüdisch. (LEVINAS 1988:61f.)

Um das Wesen des Gedichts zu ergründen, berief sich Levinas auf Der Meridian. Jetzt nennt er Gespräch im Gebirg und Engführung - zwei Texte, von denen jeder auf seine eigene Art über das Schicksal der Juden spricht: sowohl darüber, was während des Zweiten Weltkrieges geschah, als auch über das ununterbrochene Unterwegssein, dessen Ursache in der Unmöglichkeit liegt, den eigenen Platz zu finden, einen Ort, wo man zu Hause ist. Nach Levinas sind Celans Texte nicht nur eine Botschaft an die Juden, sondern haben einen universellen Charakter. 
Katarzyna Dzikowska

Zweifellos hatte Israels Passion unter Hitler [...] in den Augen des Dichters eine allgemeinmenschliche Bedeutung, da das Judentum eine extreme Möglichkeit oder Unmöglichkeit - der Menschheit darstellt und hier mit der Naivität des Botschafters oder Hirten des Seins gebrochen wird. Ein Aufplatzen der Welt, die, um die Nacht zu verbringen, keinen Ruheplatz mehr, sondern nur Steine bietet, gegen die der Stock des Wanderers stößt, der in metallischer Sprache erklingt. (LEVINAS 1988:62)

Es geht also nicht nur um die Dichtung, aber es geht auch nicht nur um das Bedenken des jüdischen Schicksals. In den Texten von Celan findet Levinas Argumente gegen die alte Denkweise - gegen eine Rationalität, die ,ausschließlich an das vom Wort getragene Sein, die an das Ausgesagte des Aussagens“" gebunden ist. Er findet dort auch eine Forderung danach, eine wahre Sprache zu finden.

Oberhalb und jenseits dieses Schweigens und dieser Bedeutungslosigkeit einer Erdfalte, die Berg heißt, und um das Geräusch des an den Stein schlagenden Stabes und die Fortsetzung dieses Geräuschs durch die Felsen zu unterbrechen, muß es - gegen , die Sprache, die hier gilt ${ }^{\star}$ - ein wirkliches Sprechen geben. (LEVINAS 1988:63)

\section{Literatur und Philosophie}

Angesichts der dargestellten Ausführungen, die grundsätzlich den Gedanken folgten, die Emmanuel Levinas in seinem Essay über die Dichtung von Paul Celan formulierte, sollte man auch die Frage nach der grundlegenden Meinung Levinas' über den Stellenwert eines literarischen Werkes wagen. Welche Bedeutung kann eine literarische Äußerung, die ihrem Wesen nach durch Fiktionalität und Distanz gekennzeichnet ist, für eine Philosophie haben, deren Fundament die Begegnung mit dem Antlitz des Menschen bildet? In seinem Essay aus dem Jahre 1948 Die Wirklichkeit und ihr Schatten betrachtete Levinas die Literatur als einen Teil der Kunst, die sich in einer verantwortungslosen Welt bewegt.

Kunst bringt die Dunkelheit des Fatums in die Welt, aber vor allem die Unverantwortlichkeit, die einen umschmeichelt wie die Anmut und die Leichtigkeit. Sie befreit. Wenn man einen Roman schreibt oder genießt und ein Gemälde malt oder Gefallen an ihm findet - dann geht es da nicht mehr um ein Begreifen, dann soll man auf die Anstrengung der Wissenschaft, der Philosophie und des Handelns verzichten. Sprechen Sie nicht! Grübeln Sie nicht! Bewundern Sie still und in aller Ruhe! - so lauten die Ratschläge einer Weisheit, die sich mit dem Schönen zufrieden gibt. Wo immer sie in Erscheinung tritt, wird die Magie als des Teufels betrachtet, innerhalb der Dichtung aber stößt sie auf eine Toleranz, die unbegreif- 
lich ist. Man rächt sich an der Schlechtigkeit, indem man eine Karikatur von ihr anfertigt, die sie zwar ihrer Wirklichkeit beraubt, sie aber nicht zerstört; man beschwört die bösen Mächte, indem man die Welt mit Idolen überschwemmt, die zwar einen Mund haben, aber nicht mehr sprechen. Als ob das Lächerliche etwas aus der Welt schaffen würde, als ob durch Lieder alles wirklich zu einem Ende kommen würde. [...] Die Welt, die vollendet werden will, wird durch die eigentliche Vollendung ihres Schattens ersetzt. Und damit meinen wir nicht die Interesselosigkeit der reinen Betrachtung, sondern die Verantwortungslosigkeit. (LEVINAS 2006:122)

Das ist eine vernichtende Kritik. Der Vorwurf der Verantwortungslosigkeit ist im Munde von Levinas einer der schwerwiegendsten Vorwürfe. Wenn man bei diesen Feststellungen bleiben möchte, so müsste man sagen, ein verantwortungsbewusster Mensch solle weder mit der Kunst noch mit der Literatur seine Zeit vergeuden. Eine solche Schlussfolgerung erscheint jedoch voreilig, vor allem wenn man all jene Äußerungen von Levinas bedenkt, in denen er mit großer Achtung über die Literatur sprach. In einem Gespräch mit Philipp Nemo im Jahre 1981 beantwortete er die Frage nach dem Beginn des Denkens folgendermaßen:

Vermutlich beginnt es mit Traumatismen oder Tastversuchen, denen man nicht einmal verbalen Ausdruck verleihen kann: eine Trennung, eine Gewaltszene, ein plötzliches Bewußtsein der Monotonie der Zeit. Mit der Lektüre von Büchern nicht unbedingt philosophischer Art - werden diese ursprünglichen Schockerlebnisse zu Fragen und Problemen, geben zu denken. Die Rolle der jeweiligen nationalen Literatur kann hier sehr bedeutend sein. Nicht, daß man in ihr Wörter lernt, aber man erlebt in ihr , das wahre Leben, das abwesend ist", das jedoch gerade nicht mehr utopisch ist. Ich denke, man unterschätzt in der großen Furcht vor allem Bücherartigen den , ontologischen' Bezug des Menschlichen zum Buch, das man als Informationsquelle betrachtet oder als ,Werkzeug' des Lernens, als Lehrbuch, während es doch eine Art und Weise unseres Seins darstellt. In der Tat bedeutet Lesen, sich über dem Realismus - oder der Politik - unserer Sorgen um uns selbst aufzuhalten, ohne indessen zu den guten Absichten unserer schönen Seele oder zur normativen Idealität dessen, was ,sein muß`, zu gelangen. In diesem Sinn wäre die Bibel für mich das Buch schlechthin. (LEvinAs 1996a:13f.)

Man könnte sich natürlich mit der Feststellung zufriedengeben, der Text Die Wirklichkeit und ihr Schatten habe mit der Zeit an Aktualität verloren, Levinas' Meinung über die Kunst und Literatur habe sich im Laufe der Jahre verändert. Dies wäre aber eine unberechtigte, vereinfachende Behauptung. Davon, dass der Text aus dem Jahre 1948 von seinem Autor als eine immer noch gültige Äußerung betrachtet wird, zeugen erstens seine Neuauflage in den Jahren 1982 und 1994 und zweitens das Faktum, dass sich Levinas auf diesen Text in seinem Werk Jenseits des Seins oder anders als Sein geschieht bezieht, wenn 
er von der Idolatrie des Schönen schreibt. Um den Text von 1948 richtig zu verstehen, darf man nicht die Äußerungen, die den Wert der Kunst und der Literatur negieren, aus dem Kontext des ganzen Textes lösen. Man darf vor allem nicht den abschließenden Abschnitt übersehen, in dem Levinas die Interpretation des (Kunst-)Werkes fordert. Wenn man die Kunst und die Literatur nur in ästhetischen Kategorien oder als Quelle des Vergnügens betrachtete, dann würden sie im Bereich der Gleichgültigkeit und Verantwortungslosigkeit bleiben. In seinem Essay De l'oblitération. Entretien avec Françoise Armengaud à propos de l'œuvre de Sosno (LEVINAS 1990) wiederholt Levinas diese Überzeugung, wenn er von den Grenzen einer ,ästhetischen Zivilisation“ spricht, die keine Worte mehr kennt, um gegen das Böse zu protestieren, und dem Leid der Welt gegenüber gleichgültig bleibt. Dank der Kritik, schrieb Levinas im Jahre 1948, wird das Werk jedoch erneut in die menschliche Welt zurückgeholt. Die Kritik führe die Dimension der Relation zu einem anderen Menschen ein. Man könne wohl sagen, durch die Interpretation gewinne das Werk die Dimension der Verantwortung, oder mindestens seien das Werk und sein Autor einem Urteil in Bezug auf die Verantwortlichkeit unterzogen (LEVINAS 2006:123f.).

Von der Bedeutung für das geistige Leben des Menschen, die Levinas der Dichtung beimaß, zeugen viele Hinweise auf diesen Bereich der menschlichen Aktivität, die der aufmerksame Leser in Jenseits des Seins oder anders als Sein geschieht finden wird. Die Termini ,Poesie‘, ,Epik‘ und ,Lyrik‘ erscheinen dort in mehreren Kontexten. Das zeugt davon, dass sie zum festen philosophischen Vokabular von Levinas gehören und für seine Denkweise wichtig sind. Schon auf den ersten Seiten von Jenseits des Seins oder anders als Sein geschieht verleiht er seiner Unsicherheit oder sogar seinem Zweifel Ausdruck, dass seine Studie ,vielleicht eine noch recht narrative, recht epische Art zu reden“ sei (LEVINAS 1998:47). An einer anderen Stelle wird er schreiben: „Erst im Gesagten, im Epos des Sagens, wird selbst die Diachronie der Zeit synchronisiert zu erinnerbarer Zeit, wird sie Thema." (LEVINAS 1998:94) Und wiederum:

Die Nähe ist eine Differenz [...] widerständig gegenüber der Erinnerung, die die Phasen einer Vergangenheit synchronisiert. Das Nicht-Darstellbare [l'inénarrable] - verliert doch der Andere in der Darstellung [dans la narration] sein Gesicht als Nächster. Unbeschreibbare Beziehung, im buchstäblichen Sinne des Wortes: unverwandelbar in Geschichte, irreduzibel auf die Gleichzeitigkeit des Geschriebenen, auf die ewige Gegenwart des Geschriebenen, das Ergebnisse verzeichnet oder präsentiert. (LEVINAS 1998:361) 
Man bemerkt in diesen Äußerungen ein prinzipielles Misstrauen dem Epischen gegenüber. Zum Wesen der Epik gehört doch, dass sie von etwas berichtet, was sich schon früher ereignet hat, was also nicht mehr da ist. Der Eindruck, man nehme an dem erzählten Geschehen teil, ist eine Täuschung. Levinas ordnet das Epische der Sphäre des Gesagten zu. ,Sagen ' (le Dire) und ,Gesagtes“ (le Dit) gehören zu den Grundbegriffen, die für das Verständnis von Jenseits des Seins oder anders als Sein geschieht unentbehrlich sind. Obwohl sie einigermaßen an die Sprache gebunden sind, erschöpft sich ihre Bedeutung nicht in dem Bereich des Sprachlichen. Eigentlich findet nur das ,Gesagte" einen sprachlichen Ausdruck als eine Artikulation von bestimmten Inhalten, als Erzählung, als philosophischer Diskurs. Der Begriff bedeutet jedoch nicht (zumindest nicht hauptsächlich) einen Sprechakt, sondern eine Situation und eine Haltung, die dem ,Sagen' gegenüber sekundär ist:

Sagen heißt, sich dem Anderen [frz.: le prochain, dem Nächsten] annähern, ihm ,Bedeutsamkeit übertragen'. Was sich nicht in ,Sinnstiftung' erschöpft, wie sie als Erzählzusammenhang dem Gesagten eingeschrieben ist. Als dem Anderen übertragene Bedeutsamkeit, übertragen vor aller Objektivierung, ist das Sagen-im-eigentlichen-Sinne keine Ausstellung von Zeichen. (LEvinAS 1998:116)

An einer anderen Stelle wird es Levinas noch deutlicher formulieren: „Die Verantwortung für den Anderen - ist genau ein solches Sagen vor allem Gesagten." (LEVINAS 1998:107) Zwischen dem Sagen und dem Gesagten herrscht eine nie aufzulösende Spannung, die sich aus einer gewissen Abhängigkeit des Sagens von dem Gesagten herleitet: „Man kann aber auf diese Bedeutung des Sagens - Verantwortung und Stellvertretung - nur zurückgehen vom Gesagten her und von der Frage ,Was hat es auf sich mit...?', die schon mit dem Gesagten, in dem alles sich zeigt, gegeben ist.“ (LEVINAS 1998:108f.) Wenn man zu den Fragen zurückkehrt, die an das Wesen des literarischen Werkes geknüpft sind, so kann man feststellen, dass dieses ,Zurückgehen vom Gesagten her“ an die interpretatorische Arbeit erinnert, wo man durch das Wortmaterial hindurch an die eigentliche Bedeutung des Textes gelangt. Bei Levinas selbst findet man die Bestätigung einer solchen These, wenn er nicht nur von der Möglichkeit, sondern von der Notwendigkeit eines solchen Vorgehens schreibt: „Aber ist es notwendig und ist es überhaupt möglich, daß das Sagen von diesseits her thematisiert wird, daß es sich manifestiert, daß es in Sätze und Bücher eingeht? Es ist in der Tat notwendig." (LEVINAS 1998:107) Woher also stammt ein solches Misstrauen dem Epischen gegenüber? An dieser Stelle muss man hervorheben, dass Levinas den Begriff des Epischen und der Epik in einem Sinne gebraucht, der sich nicht streng an die Literaturwissenschaft hält. Das Epische bedeutet nicht nur eine bestimmte Art der sprachlichen Mitteilung, 
sondern eine menschliche Handlung und Haltung dem anderen Menschen gegenüber. Einer solchen Art des Redens, einer solchen Haltung gegenüber bleibt Levinas kritisch. Man müsste das Problem vielleicht folgendermaßen erläutern: Das Epische bedeutet, dass der Mensch bei dem Gesagten (le Dit) stehen bleibt, sich damit begnügt und annimmt, dass es nichts anderes mehr gebe. Vom Epischen unterscheidet Levinas eindeutig das Lyrische. Er versteht die Lyrik als Gedicht - aber man muss schon jetzt sagen, dass der Versuch einer Analyse seiner Äußerungen zu diesem Thema oder der Formulierung eindeutiger Schlussfolgerungen eine sehr schwierige Aufgabe darstellt. Einerseits findet man Feststellungen, in denen die Lyrik als ein Gesagtes betrachtet wird:

Gewiß läßt sich das Gesagte als vorgängig zur Kommunikation und zur intersubjektiven Vergegenwärtigung des Seins verstehen. Das Sein hätte dann eine Bedeutung - das heißt es manifestierte sich - schon als in der schweigenden und nicht-menschlichen Sprache evoziertes Sein, evoziert durch die Stimme des Schweigens - im Geläut der Stille [dt. im Original], in der Sprache, die früher als die Menschen spricht und die das esse ipsum beherbergt, einer Sprache, die durch das Gedicht in menschliche Worte gesetzt wird. [...] Im Gedicht den Anklang des Seins, der das Gedicht ermöglicht, zum Erklingen bringen, hieße ein Gesagtes zum Erklingen bringen. (LEVINAS 1998:296f.)

Andererseits schreibt Levinas: „Aber ist nicht die Poesie der Welt schon vor der Wahrheit der Dinge und untrennbar von der Nähe par excellence, der des Nächsten, oder von der Nähe des Nächsten par excellence?“ (LEVINAS 1998: 173, Anmerkung 10) Und an einer anderen Stelle: „Als Zeichen, das von dieser Bedeutung als Zeichen gegeben wird, zeichnet die Nähe zugleich den Tropos der Lyrik: lieben darin, daß man die Liebe dem Geliebten sagt - Liebeslied, Möglichkeit der Dichtung, der Kunst.“ (Levinas 1998:318, Anmerkung 10) Dieses Zeichengeben ist keine Vermittlung von Informationen, was die Domäne des Gesagten ist, sondern ein Sagen,

[...] das seine Offenheit offen hält [...] Sagen, das nichts anderes sagt als ebendas Sagen, ohne es zu thematisieren, vielmehr indem es das Sagen immer noch weiter aussetzt: Sagen heißt so Zeichengeben von nichts anderem als von ebenjener Zeichenhaftigkeit, jener Bedeutsamkeit der Ausgesetztheit. [...] es heißt Zeichen geben, indem man sich zum Zeichen macht, ohne sich auszuruhen in der eigenen Zeichengestalt. (LEVINAS 1998:313)

Die Nähe, von der Levinas schreibt, ist als die Verantwortlichkeit für den Anderen zu verstehen.

Emmanuel Levinas und Paul Celan sind höchstwahrscheinlich einander nie persönlich begegnet. Man darf hier jedoch von einer anderen Dimension der 
Begegnung sprechen. Und es geht gar nicht darum, dass sie jeweils die Texte des anderen hätten lesen sollen. $\mathrm{Zu}$ einer solchen Begegnung ist es dann auch nur durch Levinas gekommen. Celan besaß zwar in seiner Bibliothek einige Bücher von Levinas, da es aber in ihnen an jeglichen Spuren der Lektüre fehlt (Celan hatte die Gewohnheit, bei der Lektüre Anmerkungen und Unterstreichungen zu machen), in manchen gar nicht alle Blätter aufgeschnitten wurden, kann man vermuten, dass sie kein größeres Interesse bei Celan geweckt haben. ${ }^{2}$ Es lässt sich schwer sagen, warum. Um so interessanter erscheint die Feststellung einer deutlichen Nähe ihres Denkens. Die oben durchgeführten Analysen sollten als eine erste Annäherung an das Problem verstanden werden und die Notwendigkeit weiterer Studien erweisen. Eine gewisse methodologische Schwierigkeit ergibt sich aus dem Faktum, dass man es mit verschiedenen Diskursen zu tun hat: von einer philosophischen Abhandlung bis zum lyrischen Gedicht. Das ist aber mit Sicherheit keine unüberwindbare Schwierigkeit. Einen Hinweis gab Celan selbst, indem er von sich selbst behauptete, er würde einen Kampf um eine Poesie führen, die mit der Wahrheit solidarisch ist. $^{3}$ In den Aufzeichnungen, die nach seinem Tode auf dem Schreibtisch gefunden wurden, gab es einen Briefentwurf an Martin Heidegger. Celan schrieb: ,[...] daß Sie durch Ihre Haltung das Dichterische und, so wage ich zu vermuten, das Denkerische, in beider ernstem Verantwortungswillen, entscheidend schwächen“ (CELAN / CELAN-LESTRANGE 2001:494).

Den Vorwurf, den Celan an Heidegger richtete, kann man auch als ein Bekenntnis des Dichters zu einer Poesie verstehen, die der Forderung nach Verantwortung verpflichtet sein muss. „Ich bin du, wenn ich ich bin“ - diese Worte aus Celans Gedicht Lob der Ferne zitierte Levinas an zwei wichtigen Stationen seines Denkens. Es gibt mehrere Möglichkeiten, diesen Vers zu lesen. Als: ich bin du erst, wenn ich ich bin. Oder: immer wenn ich ich bin, bin ich du. Oder: um du zu sein, muss ich ich werden. Um zu begreifen, wer du bist, muss ich ich werden. Vielleicht kann bei der Lektüre eines solchen Verses

2 In Celans Privatbibliothek befinden sich folgende Texte von Levinas: Die Bücher Difficile liberté (1963), De l'existence à l'existant (1947), En découvrant l'existence avec Husserl et Heidegger (1949) und die Beiträge Enigme et phénomène (1965), L'autre dans Proust (1947), Sans identité (1970); vgl. Celan (2004: 497f.). Auch bei der Lektüre des Buches von von Jacques Derrida L'écriture et la différence, das Celan zum Teil sehr aufmerksam studierte, hatte er höchstwahrscheinlich das Levinas gewidmete Kapitel übersprungen (CELAN 2004:480-484).

3 Im Brief an Petre Solomon: Je mène, depuis longtemps, un combat, celui de la poésie solidaire de la vérité. Zit. nach FELSTINER (2000:292). 
die Philosophie von Emmanuel Levinas behilflich sein. Vielleicht nicht nur bei diesem Vers. Vielleicht nicht nur bei Celan.

\section{Literatur}

Böttiger, Helmut (1996): Orte Paul Celans. Wien.

Celan, Paul (1971): Strette. Poèmes suivis de Méridien et d'Entretiens dans la montagne. Aus dem Deutschen von André du Bouchet, Jean-Pierre Burgart, Jean Daive, John E. Jackson. Paris.

- (1986): Gesammelte Werke. Bd. 3. Frankfurt (M.).

- (1999): Der Meridian. Endfassung - Entwürfe - Materialien. Frankfurt (M.).

- (2004): La bibliothèque philosophique - Die philosophische Bibliothek. Hrsg. von Alexandra Richter, Patrik Alac, Bertrand Badiou. Paris.

Celan, Paul / Celan-Lestrange, Gisèle (2001): Briefwechsel. Bd. 2. Hrsg. von Bertrand Badiou und Eric Celan. Frankfurt (M.).

Chalfen, Israel (1983): Paul Celan. Eine Biographie seiner Jugend. Frankfurt (M.).

Felstiner, John (2000): Paul Celan. Aus dem Amerikanischen von Holger Fliessbach. München.

LEvinAs, EmMANUEL (1988): Eigennamen. Aus dem Französischen von Frank Miething. München/Wien.

- (1990): De l'oblitération. Entretien avec Françoise Armengaud à propos de l'œuvre de Sosno. Paris.

- (1996): Ethik und Unendliches. Gespräche mit Philippe Nemo. Aus dem Französischen von Peter Engelmann. Wien.

- (1996a): Gott, der Tod und die Zeit. Aus dem Französischen von Astrid Nettling und Ulrike Wasel. Wien.

- (1998): Jenseits des Seins oder anders als Sein geschieht. Aus dem Französischen von Thomas Wiemer. München.

- (2003): Paul Celan. De l'être à l'autre. Montpellier.

- (2006): Die Wirklichkeit und ihr Schatten. In: LevinAs, EMmANuEL: Die Unvorhersehbarkeiten der Geschichte. Aus dem Französischen von Alwin Letzkus. München.

Malka, SAlomon (2003): Emmanuel Levinas. Eine Biographie. Aus dem Französischen von Frank Miething. München. 